\title{
Non-mass Enhancement in Breast MRI: Characterization with BI-RADS Descriptors and ADC Values
}

\author{
Wolfgang Buchberger ${ }^{1,3 *}$, Willi Oberaigner ${ }^{2}$, Christian Kremser ${ }^{3}$, Kurt Gautsch ${ }^{4}$, \\ Uwe Siebert ${ }^{2,5,6}$ \\ ${ }^{1}$ Institute of Quality and Efficiency in Medicine, UMIT - University for Health Sciences, Medical Informatics and Technology, A-6060 \\ Hall in Tirol, Austria. \\ ${ }^{2}$ Institute of Public Health, Medical Decision Making and HTA, UMIT - University for Health Sciences, Medical Informatics and \\ Technology, A-6060 Hall in Tirol, Austria. \\ ${ }^{3}$ Department of Radiology, Medical University of Innsbruck, A-6020 Innsbruck, Austria. \\ ${ }^{4}$ Radiology Institute Dr. Schöpf, A-6500 Landeck, Austria. \\ ${ }^{5}$ Harvard T. H. Chan School of Public Health, Center for Health Decision Science and Department of Health Policy and Management, \\ Boston, MA 02115, United States. \\ ${ }^{6}$ Massachusetts General Hospital, Harvard Medical School, Institute for Technology Assessment and Department of Radiology, Boston, \\ MA 02114, United States.
}

Received 20 February 2021; Revised 24 March 2021; Accepted 05 April 2021; Published 01 June 2021

\begin{abstract}
Objectives: The purpose of this study was to assess the accuracy of contrast-enhanced magnetic resonance imaging and diffusion-weighted imaging in distinguishing benign from malignant non-mass-like breast lesions. Methods: 103 lesions showing non-mass-like enhancement in 100 consecutive patients were analyzed. Distribution, internal enhancement patterns, and contrast kinetic curve patterns were classified according to the BI-RADS lexicon. Apparent diffusion coefficient (ADC) values were obtained from manually placed regions of interest (ROIs) on diffusion-weighted images. The optimal ADC value threshold for the distinction between benign and malignant lesions was determined by ROC analysis. Univariate and multivariate analyses were performed to identify independent predictors of malignancy, and the probability of malignancy was calculated for various combinations of findings. Histological diagnosis obtained by means of core needle biopsy was used as gold standard. Results: According to the univariate and multivariate analysis, odds ratios for malignancy were significantly elevated for clumped or clustered ring internal enhancement and low ADC values ( $\mathrm{p}$ 0.001), whereas distribution patterns and contrast kinetic patterns were not significantly correlated with benignity or malignancy. In non-mass lesions with homogeneous or heterogeneous internal enhancement and ADC values greater than $1.26 \times 10^{-3} \mathrm{~mm}^{2} / \mathrm{s}$, no malignancy was detected, while all other combinations of findings had a probability of malignancy ranging from 22.2 to $76.6 \%$. Conclusions: A combination of BI-RADS descriptors of internal enhancement and ADC values is useful for the differential diagnosis of lesions showing non-mass enhancement. Lesions with homogeneous or heterogeneous enhancement and high ADC can be followed up, while all other lesions should be biopsied.

Keywords: Breast; Magnetic Resonance Imaging; Non-mass Enhancement; Diffusion-weighted Imaging.
\end{abstract}

\section{Introduction}

Breast MRI is a highly sensitive method for the early detection and characterization of breast cancer [1-3]. However, distinguishing benign from malignant breast lesions based on MR imaging characteristics remains difficult

* Corresponding author: wolfgang.buchberger@umit.at

doi http://dx.doi.org/10.28991/SciMedJ-2021-0302-1

$>$ This is an open access article under the CC-BY license (https://creativecommons.org/licenses/by/4.0/).

(C) Authors retain all copyrights. 
$[4,5]$. One attempt at improving diagnostic accuracy has been standardization of reading and reporting of breast MR examinations by the introduction of the Breast Imaging Reporting and Data System (BI-RADS) by the American College of Radiology [6, 7].

The BI-RADS lexicon distinguishes three types of breast abnormalities: mass lesions, non-mass enhancement, and foci. Mass lesions are defined as space-occupying lesions that are visible on pre- and postcontrast scans, and may displace or otherwise affect the surrounding breast tissue. In contrast, non-mass enhancement (NME) is the contrast enhancement of an area that is not a mass and may extend over small or large regions of the breast, and whose internal enhancement characteristics can be described as a pattern discrete from the normal surrounding breast parenchyma.

The underlying pathology is variable and includes invasive carcinoma, ductal carcinoma in situ (DCIS), high risk lesions such as atypical ductal hyperplasia and lobular carcinoma in situ, and benign mastopathic changes [8]. Malignancy rates of non-mass enhancement have been reported to be in the range of 39-51.7\% [9, 10]. In a review of non-palpable, mammographically occult invasive cancers detected by MRI, 57\% were cancers with non-mass enhancement [11].

Characteristics of non-mass enhancement as described in the BI-RADS lexicon, include distribution (foal, linear, segmental, regional, multiple regions, or diffuse), internal enhancement pattern (homogeneous, heterogeneous, clumped, or clustered ring enhancement), and contrast kinetic curves [6]. However, the value of BI-RADS descriptors for characterization of non-mass enhancement is significantly lower than for mass lesions [10, 12-14]. In a metaanalysis of 858 non-mass lesions, pooled estimates of sensitivity and specificity were 50 and $80 \%$, respectively [9]. This diagnostic uncertainty may lead to a significant number of unnecessary biopsies.

Diffusion weighted (DWI) imaging has been shown to be useful for the characterization of breast abnormalities Although there is only limited experience with DWI in non-mass enhancement, preliminary reports indicate that the addition of apparent diffusion coefficient (ADC) mapping to contrast-enhanced MRI could improve diagnostic accuracy [15-18]. The purpose of this study was to investigate the performance of BI-RADS descriptors combined with $\mathrm{ADC}$ values for the characterization of non-mass enhancement on contrast-enhanced MR imaging of the breast.

\section{Materials and Methods}

\subsection{Patients}

This study was approved by the ethics committee of the Medical University of Innsbruck. Patient informed consent was waived because of the retrospective design. A retrospective search of the MRI database of the Department of Radiology at the University Hospital Innsbruck was performed to identify breast MRI examinations showing nonmass enhancement between June 2016 and June 2020. A total of 123 consecutive cases of non-mass enhancement with histopathological confirmation were identified. DWI was not performed in 14 lesions. Six lesions were excluded because of technical issues with the DWI sequence, including inadequate fat suppression $(n=5)$ and motion artifacts $(\mathrm{n}=1)$. The remaining 103 non-mass enhancements in 100 women (median age 51 years; range, 21-84 years) were included in the study.

Indications for MR imaging examination included evaluation of indeterminate findings on mammography or ultrasound in 35 patients, high-risk screening in 22 patients, preoperative tumor staging in 17 patients, oncologic follow-up after breast conserving therapy in 15 patients, and clinical abnormalities in 14 patients. Clinical abnormalities included palpable lesions in eight patients and nipple discharge in six patients. The study lesions detected in the patients who underwent MR imaging for staging of otherwise detected breast cancer were additional lesions distinct from the known biopsy-proven cancer.

Histopathologic diagnosis was obtained with MR-guided vacuum-assisted core biopsy in 82 cases, with ultrasound-guided core biopsy in 19 cases and with stereotactic vacuum-assisted core biopsy in two cases. The final diagnosis was obtained by surgical excision in all malignant and high-risk lesions.

\subsection{MRI Imaging Protocol}

All patients underwent breast MRI on a 1.5 T system (Magnetom Avanto, Siemens Healthcare) with an 8-channel bilateral breast coil. To avoid artifacts due to bleeding or clip markers, all imaging was performed before any biopsy. The imaging protocol included an axial T2-weighted turbo inversion recovery sequence (TR /TE/TI, 5160/61/170 ms; flip angle, $170^{\circ}$; matrix, $336 \times 448$, slice thickness, $4 \mathrm{~mm}$; time of acquisition, 2:30 minutes) and an axial dynamic contrast-enhanced T1-weighted 3D spoiled gradient echo sequence with spectral-attenuated inversion recovery (SPAIR) fat suppression (TR/TE, 5.3/2.4 ms, flip angle, $10^{\circ}$; matrix, 422×480; FOV, 280-300 $\mathrm{cm}^{2}$; number of slices: 112; slice thickness, $1.5 \mathrm{~mm}$; time of acquisition, 87 seconds). One pre-contrast and five post-contrast acquisitions centered at 90, 180, 270, 360, and 450 seconds were acquired. For contrast-enhanced imaging, $0.1 \mathrm{mmol} / \mathrm{kg}$ body weight gadobutrol was injected intravenously with an injection rate of $2 \mathrm{ml} / \mathrm{s}$. 
DWI was performed before the dynamic contrast enhanced acquisitions, using a readout-segmented EPI diffusionweighted sequence (RESOLVE; TR/TE1/TE2, 5270/56 ms /87 ms; flip angle, $180^{\circ}$, matrix size, $216 \times 98$, pixels, field of view, $154 \times 340 \mathrm{~mm}, 28$ slices with $5 \mathrm{~mm}$-slice thickness). Diffusion gradients were applied in three directions (diffusion mode: 3-Scan Trace) with b values of 50, 400, and $800 \mathrm{~s} / \mathrm{mm} 2$. The acquisition time was 5:02 minutes.

\subsection{MR Image Interpretation}

Evaluation of MR images was done on a dedicated workstation (syngo.via, Siemens Healthcare). Post-processing included multi-planar reconstruction of contrast-enhanced images, maximum intensity projections, calculation of parametric images (maximum enhancement integral), and calculation of time-signal intensity curves.

All images were retrospectively viewed by two experienced readers (one having a 10-year experience and another having a 30-year experience in breast imaging) who made their assessment in consensus. Both readers were blinded to all additional imaging results and to clinical, follow-up, and histopathological data. Axial images and reconstructed sagittal and coronal images were used to determine the spatial distribution of enhancement. Enhancement distribution and internal enhancement patterns were classified according to the 5th edition of the BI-RADS MRI lexicon [6].

Construction of time-signal intensity curves and measurement of ADC values was done by one of the two readers. Kinetic curves were obtained from manually placed circular regions of interest (ROI) that covered the areas with the greatest degree of early enhancement. Kinetic curves were classified according to the BI-RADS MRI lexicon. Curves with a persistent delayed phase ware classified as type 1, curves with a plateau delayed phase were classified as type 2 , and curves with a washout were classified as type 3. Mean ADC values were measured on the ADC maps by using circular ROIs with a size of $10-20 \mathrm{~mm}^{2}$. The ROIs were placed in the areas of lowest ADC-values on visual assessment. At least three measurements were obtained for each lesion and the lowest mean value was selected. Partial volume effects due to surrounding tissue and necrosis were avoided as far as possible by sparing the lesion borders and obvious areas of necrosis, as shown on T2-weighted and contrast-enhanced images.

\subsection{Statistical Analysis}

For patient characteristics, numbers by benign/high-risk/malignant lesions were computed. Positive predictive values were calculated as PPV3 (i.e., the percentage of cancers in cases that underwent biopsy), as recommended by the 5th edition of the ACR BIRAS lexicon [6]. In order to determine a feasible ADC value threshold, a receiver operating characteristic (ROC) analysis was applied and the threshold was defined as the ADC value where sensitivity and specificity crossed. Comparisons of the means of two independent samples were made using the t-test for normally distributed variables and the Mann-Whitney U test for non-normally distributed variables.

For univariate analysis, odds ratios (OR) for malignant lesions compared to benign and high-risk lesions were computed for ADC, distribution of enhancement, internal enhancement, contrast kinetic curve type, tumor size, and presence of a correlate on mammography or ultrasound. We calculated $95 \%$ confidence intervals and $p$ values by applying the likelihood ratio test. For multivariate analysis, all variables tested in the univariate analysis with a $\mathrm{p}$ value $\leqslant 0.1$ were put in the model. Finally, the prediction probability for malignancy for the combinations of ADC values and internal enhancement was determined.

All statistical analysis was done using Stata Version 13.1 (StataCorp LP, 4905 Lakeway Drive College Station, TX 77845, USA). Statistical significance was established at alpha $=0.05$ (except for building the multivariate model).

\section{Results}

Pathohistological findings are summarized in Table 1. Of the 103 lesions in our study, 19 were invasive carcinomas, 21 were ductal carcinoma in situ, six were high-risk lesions (confirmed on surgical excision, without upgrade), and 57 were benign. The overall prevalence of malignancies was $38.8 \%$. The mean diameter of benign and high-risk lesions was $31 \mathrm{~mm}$ (range, 6-102 mm), and the mean diameter of malignant lesions was $29 \mathrm{~mm}$ (range, 7-70 $\mathrm{mm} ; \mathrm{p}>0.05)$.

In three benign and seven malignant lesions, microcalcifications were visible on mammography in the localization corresponding to the non-mass enhancement. In 13 benign and eight malignant lesions, an ultrasound correlate was detected either on routine sonography $(n=4)$ or on targeted second-look sonography $(n=17)$. The ultrasound findings were mass lesions $(n=16)$, clustered microcysts $(n=2)$, duct changes $(n=2)$, and architectural distortion $(n=1)$. 
Table 1. Pathohistological diagnosis in 103 non-mass lesions

\begin{tabular}{lc}
\hline \multicolumn{1}{c}{ Final diagnosis } & N lesions (percentage) \\
\hline Benign lesions & $57(55.3)$ \\
\hline Mastopathic changes & $34(33.0)$ \\
Papilloma & $8(7.8)$ \\
Fibroadenoma & $6(5.8)$ \\
Focal mastitis & $7(6.8)$ \\
Fat tissue necrosis & $2(1.9)$ \\
\hline High-risk lesions & $6(5.8)$ \\
\hline Atypical ductal hyperplasia & $4(3.9)$ \\
Lobular carcinoma in situ & $2(1.9)$ \\
\hline Malignant tumors & $40(38.8)$ \\
\hline DCIS & $21(20.4)$ \\
Invasive carcinoma NST & $12(11.7)$ \\
Invasive lobular carcinoma & $7(6.8)$ \\
\hline
\end{tabular}

MR imaging characteristics in the 103 non-mass-like lesions are summarized in Table 2. The most common distribution pattern was linear $(40.8 \%$; 42/103), followed by focal $(29.1 \% ; 30 / 103)$ and regional $(16.5 \% ; 17 / 103)$. The most common internal enhancement pattern was clumped $(42.7 \%$; 44/103), followed by homogeneous $(34.0 \%$; $35 / 103)$ and heterogeneous (17.5\%; 18/103). Clustered ring enhancement was present in six cases, including two lobular invasive carcinomas, one atypical ductal hyperplasia, one lobular carcinoma in situ and two benign fibrocystic changes.

Table 2. Imaging characteristics of 103 non-mass lesions

\begin{tabular}{|c|c|c|c|c|c|}
\hline Descriptor & $\begin{array}{l}\text { Benign } \\
(\mathrm{n}=57)\end{array}$ & $\begin{array}{l}\text { High Risk } \\
\quad(n=6)\end{array}$ & $\begin{array}{c}\text { Malignant } \\
(\mathrm{n}=\mathbf{4 0})\end{array}$ & $\begin{array}{c}\begin{array}{c}\text { Total } \\
(\mathbf{n}=103)\end{array} \\
\end{array}$ & $\begin{array}{c}\text { PPV3 } \\
(\%)\end{array}$ \\
\hline \multicolumn{6}{|l|}{ Internal enhancement } \\
\hline Homogeneous & 32 & 1 & 2 & 35 & 5.7 \\
\hline Heterogeneous & 12 & 2 & 4 & 18 & 22.2 \\
\hline Clumped & 11 & 1 & 32 & 44 & 72.7 \\
\hline Clustered ring & 2 & 2 & 2 & 6 & 33.3 \\
\hline \multicolumn{6}{|l|}{ Distribution } \\
\hline Focal & 19 & 1 & 10 & 30 & 33.3 \\
\hline Linear & 21 & 1 & 20 & 42 & 47.6 \\
\hline Segmental & 6 & 1 & 6 & 13 & 46.2 \\
\hline Regional & 10 & 3 & 4 & 17 & 23.5 \\
\hline Diffuse & 1 & & & 1 & 0.0 \\
\hline \multicolumn{6}{|c|}{ Time intensity curve type } \\
\hline Type 1 & 43 & 6 & 23 & 72 & 31.9 \\
\hline Type 2 & 13 & & 12 & 25 & 48.0 \\
\hline Type 3 & 1 & & 5 & 6 & 83.3 \\
\hline \multicolumn{6}{|l|}{ ADC value } \\
\hline$<1.26 \times 10^{-3} \mathrm{~mm}^{2} / \mathrm{sec}$ & 14 & 2 & 29 & 45 & 64.4 \\
\hline$\geq 1.26 \times 10^{-3} \mathrm{~mm}^{2} / \mathrm{sec}$ & 43 & 4 & 11 & 58 & 19.0 \\
\hline
\end{tabular}

Of 21 DCIS, 12 showed a linear clumped enhancement, three a segmental clumped enhancement, two a regional clumped enhancement, two a focal heterogeneous enhancement, one a linear homogeneous enhancement, and one a focal homogeneous enhancement. Of 19 invasive carcinomas, seven showed a linear clumped enhancement, five a focal clumped enhancement, two a segmental clumped enhancement, two a focal heterogeneous enhancement, and one each a regional clumped enhancement, a segmental enhancement with clustered ring enhancement and a regional enhancement with clustered ring enhancement. 


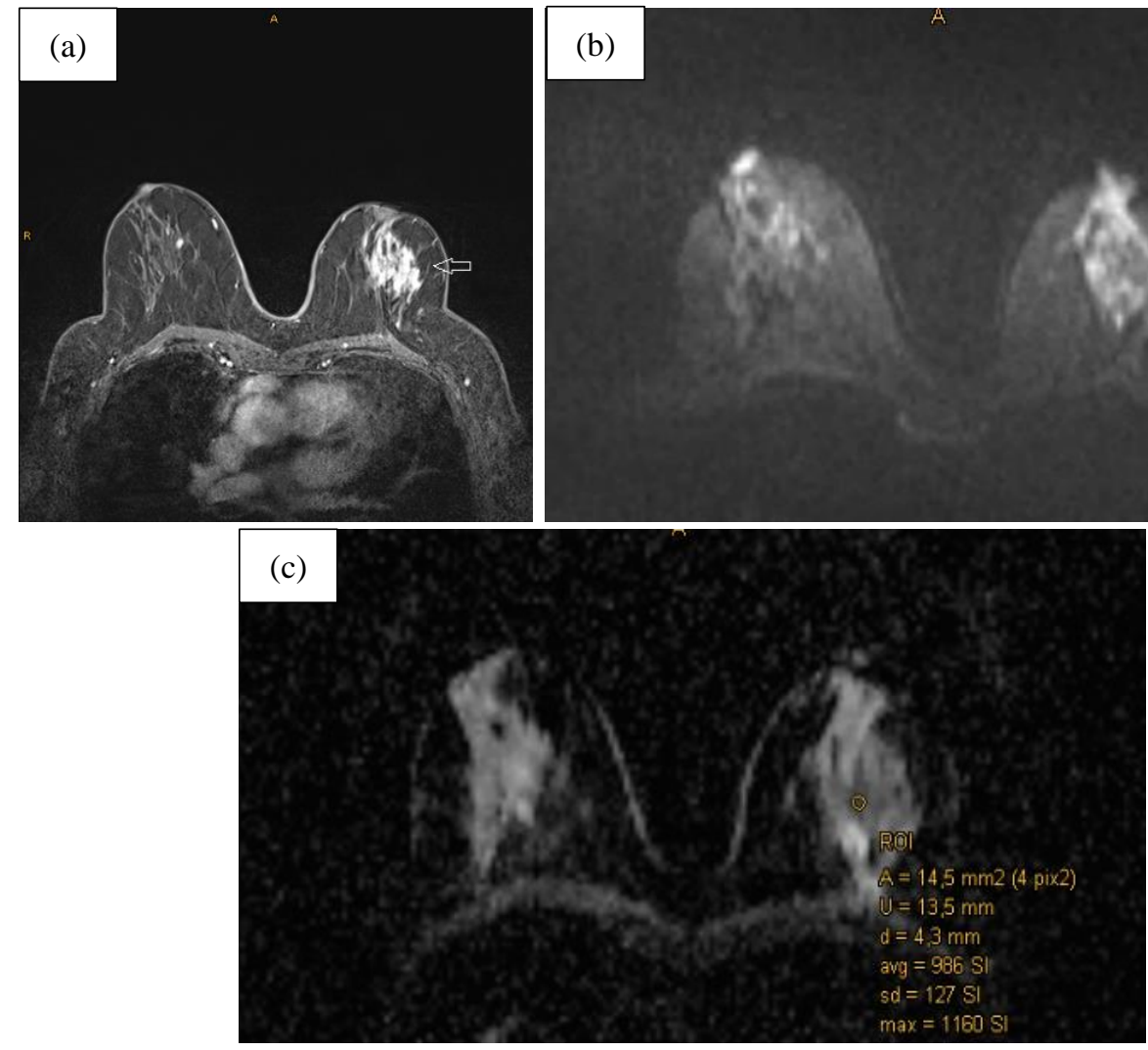

Figure 1. MRI of the breast: 64-year-old woman with lobular invasive carcinoma. Axial T1-weighted contrast-enhanced image (a) shows regional heterogeneous enhancement in left breast (arrow). Corresponding diffusion-weighted image $(b=$ $800 \mathrm{~s} / \mathrm{mm}^{2}$ ) (b) and ADC map (c) show restricted diffusion with low ADC values (arrow).

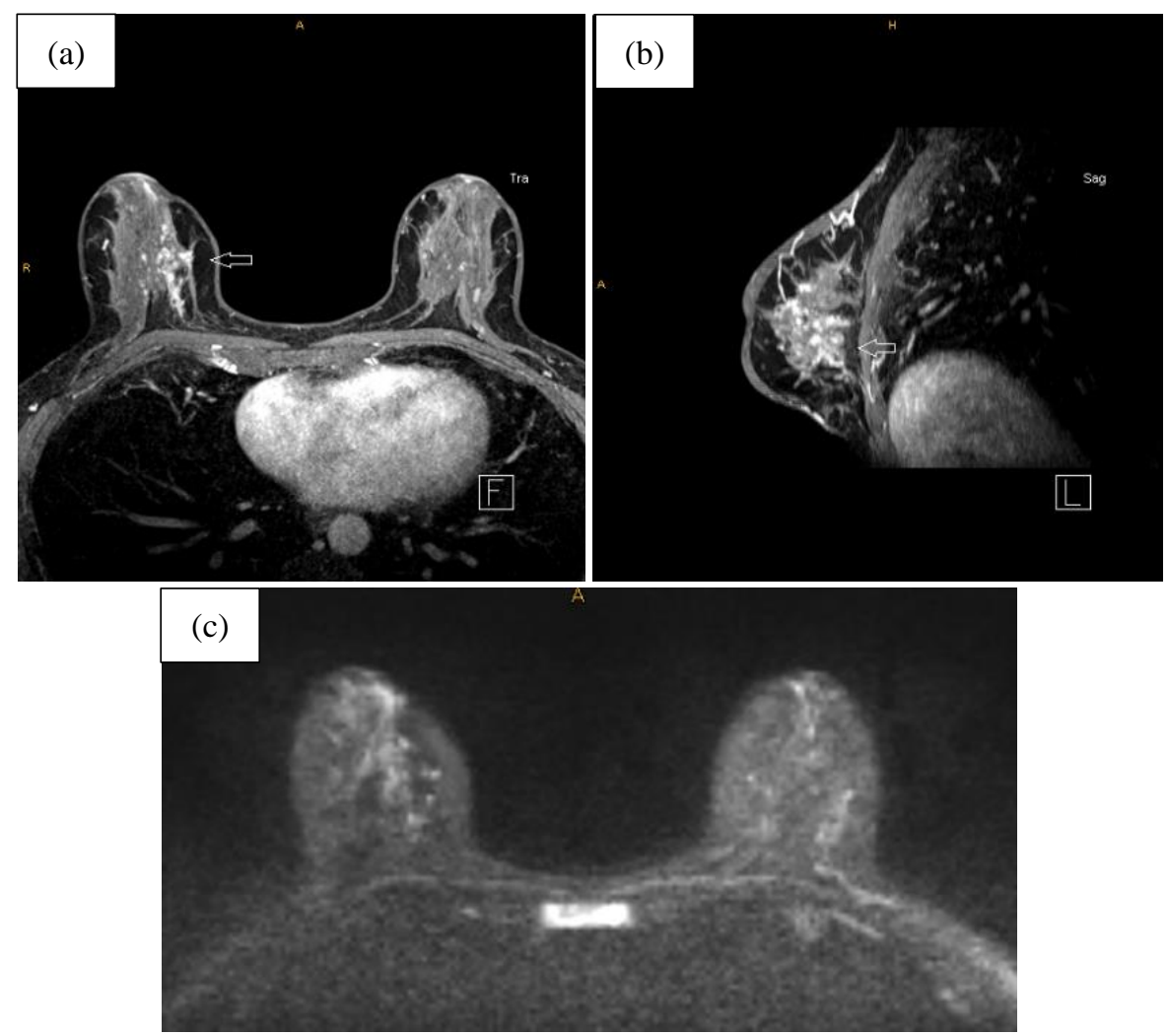

Figure 2. MRI of the breast: 45year-old woman with atypical ductal hyperplasia. Axial (a) and sagittal reconstructed (b) contrast-enhanced T1-weighted images show linear clumped enhancement in right breast (arrow). Corresponding diffusionweighted image $\left(b=800 \mathrm{~s} / \mathrm{mm}^{2}\right)$ (c) shows no evidence of restricted diffusion. 
Of the distribution patterns, the highest positive predictive value was found in linear distribution (PPV3, 47.6\%), followed by segmental distribution (PPV3, 46.2\%). The internal enhancement patterns with the highest positive predictive values were clumped enhancement (PPV3, 72.7\%) and clustered ring enhancement (PPV3, 33.3\%). The positive predictive value for detection of malignancy was $31.9 \%$ (23/72) for a contrast kinetic curve showing a persistent delayed phase, $48 \%$ (12/25) for a curve showing a plateau phase, and $83.3 \%(5 / 6)$ for a curve showing a washout delayed phase. The six cases with a washout curve were three invasive carcinomas, two high-grade DCIS and one papilloma.

The mean ADC value of benign and high-risk lesions was $1.40 \times 10^{-3} \mathrm{~mm}^{2} / \mathrm{s}$ (range, $0.65-2.51 ; \mathrm{SD}, 0.29$ ), while the mean $\mathrm{ADC}$ value of malignant lesions was $1.10 \times 10^{-3} \mathrm{~mm}^{2} / \mathrm{s}$ (range, 0.70-1.92; SD, 0.29); the difference was statistically significant $(\mathrm{p}<0.001)$. ROC curve analysis revealed the most effective ADC value threshold as $1.26 \times$ $10^{-3} \mathrm{~mm}^{2} / \mathrm{s}$ (Figure 3). Compared to this threshold value, 29 of 40 malignant lesions (72.5\%) and 16 of 19 invasive carcinomas $(84.2 \%)$ had a lower ADC.

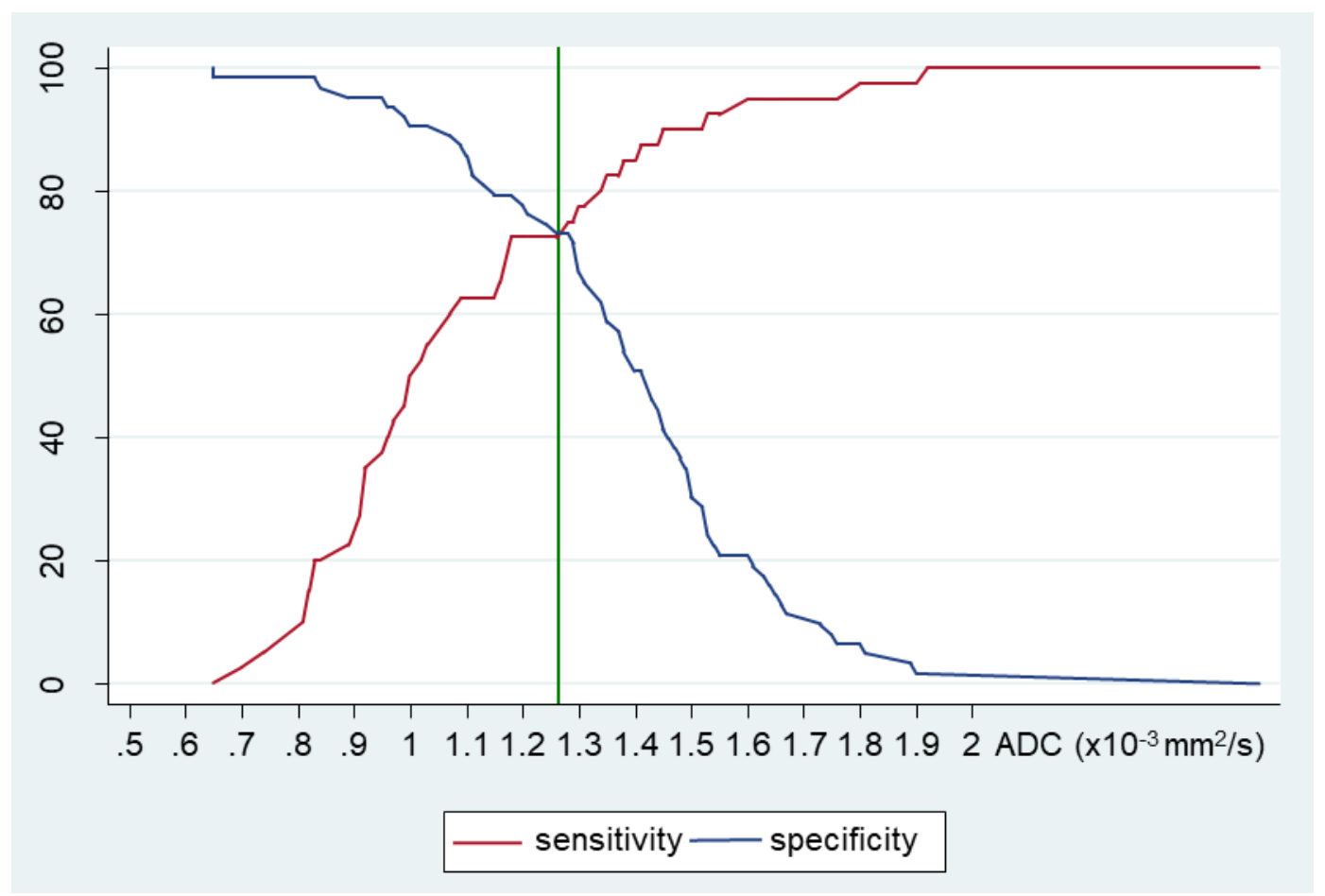

Figure 3. Results of the ROC-based positive test at different ADC values. The optimum cut-off level is determined to be $1.265 \times 10^{-3} \mathrm{~mm}^{2} / \mathrm{s}$

For univariate and multivariate analysis of MR imaging characteristics, linear and segmental enhancement, and regional and diffuse enhancement were combined in one group. In addition, clustered ring enhancement was combined with clumped enhancement. For the assessment of the influence of lesion size, lesions were divided into those under $25 \mathrm{~mm}$ and over $25 \mathrm{~mm}$. The presence of a correlate on mammography or ultrasound was also included in the analysis. According to the univariate analysis, odds ratios were significantly elevated $(\mathrm{p}<0.05)$ for clumped or clustered ring enhancement, type 3 contrast kinetic curves, the presence of microcalcifications on mammography, and ADC values less than $1.26 \times 10^{-3} \mathrm{~mm}^{2} / \mathrm{s}$ (Table 3). Multivariate analysis confirmed clumped internal enhancement and low ADC values as independent indicators of malignancy (Table 4).

Table 3. Univariate analysis results

\begin{tabular}{lcc}
\hline & $\begin{array}{c}\text { Odds ratio univariate } \\
\mathbf{9 5 \%} \text { confidence interval) }\end{array}$ & $\begin{array}{c}\text { P value } \\
\text { (Likelihood ratio test) }\end{array}$ \\
\hline ADC value & & \\
\hline$\geq 1.26 \times 10^{-3} \mathrm{~mm}^{2} / \mathrm{sec}$ & Reference & $<0.001$ \\
$<1.26 \times 10^{-3} \mathrm{~mm}^{2} / \mathrm{sec}$ & $7.13(2.93-17.36)$ & $<0.001$ \\
\hline Internal enhancement & Reference & 0.093 \\
\hline Homogeneous & $35.06(7.47-164.54)$ & \\
Clumped/clustered ring enhancement & $4.71(0.77-28.77)$ & \\
\hline
\end{tabular}




\begin{tabular}{lcc}
\hline \multicolumn{1}{l}{ Distribution } & & \\
\hline Fokal & Reference & \\
Linear/segmental & $1.79(0.71-4.52)$ & 0.216 \\
Regional/diffuse & $0.57(0.15-2.19)$ & 0.415 \\
\hline Time intensity curve type & & \\
\hline Type 1 & Reference & 0.51 \\
Type 2 & $1.97(0.78-4.97)$ & 0.035 \\
Type 3 & $10.65(1.18-96.47)$ & \\
\hline Maximum diameter & Reference & 0.68 \\
\hline$\leq 26$ mm & $1.18(0.53-2.62)$ & \\
$>26$ mm & & 0.94 \\
\hline Ultrasound correlate & Reference & \\
\hline No & $0.96(0.36-2.58)$ & 0.046 \\
Yes & & \\
\hline Microcalcifications & Reference & \\
\hline No & $4.24(1.03-17.51)$ & \\
Yes & & \\
\hline
\end{tabular}

Table 4. Multivariate analysis results

\begin{tabular}{lcc}
\hline & $\begin{array}{c}\text { Odds ratio multivariate } \\
\mathbf{( 9 5 \%} \text { confidence interval) }\end{array}$ & $\begin{array}{c}\text { P value } \\
\text { (Likelihood ratio test) }\end{array}$ \\
\hline ADC High & Reference & \\
$\quad$ Low & $5.80(1.99-16.90)$ & 0.001 \\
\hline Intern. Enhancement & Reference & \\
\hline Homogeneous & $29.36(5.89--146.25)^{1)}$ & $<0.001$ \\
Clumped/clustered ring enhancement & $4.13(0.63-27.12)^{2)}$ & 0.14 \\
\hline
\end{tabular}

Note: $\mathrm{ADC}$ high $=\mathrm{ADC} \geq 1.26 \times 10^{-3} \mathrm{~mm}^{2} / \mathrm{s} ; \mathrm{ADC}$ low $=\mathrm{ADC}<1.26 \times 10^{-3} \mathrm{~mm}^{2} / \mathrm{s}$.

The prediction probabilities for malignancy for the combination of ADC values and internal enhancement patterns are summarized in Table 5. Non-mass-like lesions with homogeneous or heterogeneous enhancement and high ADC values were all benign. All other combinations had higher probabilities of malignancy, ranging from $22.2 \%$ for the combination of homogeneous enhancement and low ADC to $76.6 \%$ for the combination of clumped or clustered ring enhancement and low ADC.

Table 5. Prediction probabilities for malignancy for the combination of enhancement patterns and ADC values

\begin{tabular}{lccc}
\multicolumn{1}{c}{ Descriptor } & N at risk & N malignant & Probability of Malignancy \\
\hline $\begin{array}{l}\text { ADC high } \\
\text { Homogeneous enhancement }\end{array}$ & 26 & 0 & 0.000 \\
$\begin{array}{l}\text { ADC high } \\
\text { Heterogeneous enhancement }\end{array}$ & 11 & 0 & 0.000 \\
$\begin{array}{l}\text { ADC high } \\
\text { Clumped/clustered ring enhancement }\end{array}$ & 20 & 11 & 0.55 \\
$\begin{array}{l}\text { ADC low } \\
\text { Homogeneous enhancement }\end{array}$ & 9 & 2 & 0.222 \\
$\begin{array}{l}\text { ADC low } \\
\text { Clumped/clustered ring enhancement }\end{array}$ & 30 & 23 & 0.767 \\
$\begin{array}{l}\text { ADC low } \\
\text { Heterogeneous enhancement }\end{array}$ & 7 & 4 & 0.571 \\
\hline
\end{tabular}




\section{Discussion}

The results of our study indicate that internal enhancement patterns combined with ADC values are best suited for distinguishing benign from malignant non-mass enhancement. Among BIRADS descriptors for distribution of enhancement, we found the highest positive predictive values for segmental (46.2\%) and linear (47.6\%) enhancement. This is comparable to the PPV for segmental enhancement of 57.4\% found by Wilhelm et al. [10] and 67\% found by Liberman et al. [19]. Tozaki and Fukuda [20] reported a PPV of 100\% for segmental distribution, which they attributed to high-resolution imaging with multiplanar reconstructions. Reported positive predictive values for linear enhancement ranged from 31.5 to $39.7 \%$ [10, 21].

Some authors concluded that BIRADS distribution descriptors, together with other imaging features, could be used for predicting the probability of malignancy [22, 23]. However, in our study, distribution patterns were not identified as independent predictors of malignancy. Of 13 cases with segmental enhancement, three were DCIS, three were invasive carcinomas, one was atypical ductal hyperplasia, and six were benign lesions. Of 42 cases with linear enhancement, 13 were DCIS, seven were invasive carcinomas, one was atypical ductal hyperplasia, and 21 were benign lesions. The benign lesions were mainly abnormalities associated with the breast ducts, such as sclerosing adenosis $(n=4)$, papillomatosis $(n=3)$, ductectasia $(n=4)$, or galactophoritis $(n=5)$. In five cases, pathohistology showed fibrocystic changes or normal breast tissue. These results suggest that segmental or linear enhancement do not indicate malignancy, but only an abnormality associated with the galactophoric ducts.

In our study, clumped internal enhancement was a strong and independent predictor of malignancy. This is consistent with other studies, which found high positive predictive values for clumped enhancement alone or in combination with ductal or segmental enhancement [10, 19-22]. There are few studies which performed regression analysis in order to adjust for confounding factors in the statistical analysis and to identify independent predictors of malignancy in non-mass enhancement. Yabuuchi et al. [18] found segmental distribution, low ADC values and clumped internal enhancement to be the strongest indicators of malignancy. In contrast, Gutierrez et al. [14] reported that BIRADS descriptors were not significant predictors of malignancy in non-mass enhancement lesions. However, for the analysis, clumped enhancement and heterogeneous, reticular or dendritic enhancement were grouped together, making it difficult to compare their results with other studies.

Unlike in previous studies, clustered ring enhancement was a relatively rare finding in our study. Only in six of 103 lesions, including two lobular invasive carcinomas, two high-risk lesions and two benign lesions, was this pattern clearly detectable. Tozaki et al. [24] described clustered ring enhancement in $63 \%$ of malignant and $4 \%$ of benign lesions. Uematsu and Kasami [22] reported this finding as being present in 66 of 124 (53\%) cases of non-mass enhancement. However, these studies analyzed clustered ring enhancement not as a separate internal enhancement pattern, as specified in the 5th edition of the ACR BI-RADS atlas, but categorically as present or not present [6]. A recent analysis of non-mass enhancement patterns using the updated BI-RADS atlas reported a frequency of clustered ring enhancement of only $20 \%$ [21]. In addition, clustered ring enhancement can be difficult to differentiate from other internal enhancement patterns, such as heterogeneous or clumped enhancement. Data on the inter-observer agreement for the diagnosis of clustered ring enhancement are not available [25].

Many studies have assessed the effectiveness of diffusion-weighted imaging (DWI) in the differentiation of benign and malignant breast lesions. A meta-analysis of 13 studies reported an overall sensitivity and specificity of DWI of $84 \%$ and $79 \%$, respectively [26]. However, there are only few studies on the effectiveness of DWI in non-mass lesions $[16,18]$. Partridge et al. [17] and Kuhl et al. [15] found that the mean ADC was significantly lower for malignant than benign lesions for both masses and non-mass enhancement, although the difference in mean ADC of benign and malignant lesions was smaller for non-mass enhancement. In our study, a low ADC was significantly associated with malignancy.

Our ADC cutoff value of $1.26 \times 10^{-3} \mathrm{~mm}^{2} / \mathrm{s}$ was similar to that in the study of Yabuuchi et al. [18]. Other studies reported cutoff values ranging from 0.9 to $1.55 \times 10^{-3} \mathrm{~mm}^{2} / \mathrm{s}[15,17]$. The differences may be explained by different scanning parameters and ROI size and placement. To date, there is no consensus about how to choose the ROIs for ADC measurements, although ROI placement and size significantly influence ADC values [27]. Minimum and mean ADC acquired from 2D-ROIs have shown to be best suited for the differentiation of benign and malignant breast lesions [27]. Small ROIs covering the area with the lowest ADC values, as used in our study, proved to perform better than large ROIs covering the whole lesion [28, 29].

Non-mass enhancement often leads to unnecessary biopsies. In a BI-RADS descriptor study of 120 malignant and 31 benign lesions, non-mass enhancement was the major cause of false-positive findings [12]. Therefore, the primary objective of our study was to identify combinations of findings that are associated with such a low probability of malignancy that biopsy can be omitted and short-term follow-up can be performed instead. This goal could be achieved by combining internal enhancement patterns with ADC values. To our knowledge, the only study with a 
similar approach is that of Yabuuchi et al. [18]. However, they examined only 31 malignant and 14 benign lesions and did not use the classification scheme of the 5th edition of the ACR BIRADS lexicon.

Our study has some limitations. First, it is a retrospective analysis from a single institution with a limited number of cases. Second, we used only those lesions that were referred for biopsy, which could have led to selection bias due to the exclusion of benign lesions without histological diagnosis. On the other hand, the proportion of malignant lesions in our study of $37.8 \%$ was comparable to other studies [9, 10]. Finally, measurements of ADC values were done by only one reader, and inter-observer agreement was not assessed. However, in previous studies, measurements of mean ADC generally showed good inter- and intra-reader agreement, independent of the approach to placement of ROI [27, 30, 31].

\section{Conclusion}

In conclusion, a combination of BI-RADS descriptors of internal enhancement and ADC values is useful for the differential diagnosis of lesions showing non-mass enhancement. Lesions with homogeneous or heterogeneous enhancement and high ADC can be followed up, while all other lesions should be biopsied.

\section{Declarations}

\subsection{Author Contributions}

Conceptualization, W.B.; methodology, W.B. and W.O.; software, W.O.; validation, U.S.; formal analysis, W.B. and K.G.; investigation, W.B. and K.G.; resources, W.B. and U.S.; data curation, W.O.; writing-original draft preparation, W.B.; writing-review and editing, U.S.; visualization, W.B.; supervision, W.B. and U.S.; project administration, W.B. All authors have read and agreed to the published version of the manuscript.

\subsection{Funding}

The authors received no financial support for the research, authorship, and/or publication of this article.

\subsection{Ethical Approval}

The study was conducted in accordance with the Declaration of Helsinki, and the protocol was approved by the Ethics Committee of the Medical University of Innsbruck.

\subsection{Data Availability Statement}

The data presented in this study are available on request from the corresponding author. The data are not publicly available due to data protection requirements

\subsection{Conflict of Interest}

The authors declare that they have no known competing financial interests or personal relationships that could have appeared to influence the work reported in this paper.

\section{References}

[1] Morrow, M., Waters, J., \& Morris, E. (2011). MRI for breast cancer screening, diagnosis, and treatment. The Lancet, 378(9805), 1804-1811. doi:10.1016/s0140-6736(11)61350-0.

[2] Sardanelli, F., Boetes, C., Borisch, B., Decker, T., Federico, M., Gilbert, F. J., ... Wilson, R. (2010). Magnetic resonance imaging of the breast: Recommendations from the EUSOMA working group. European Journal of Cancer, 46(8), $1296-1316$. doi:10.1016/j.ejca.2010.02.015.

[3] Morris, E. A. (2007). Diagnostic Breast MR Imaging: Current Status and Future Directions. Radiologic Clinics of North America, 45(5), 863-880. doi:10.1016/j.rcl.2007.07.002.

[4] Millet, I., Pages, E., Hoa, D., Merigeaud, S., Curros Doyon, F., Prat, X., \& Taourel, P. (2012). Pearls and pitfalls in breast MRI. The British Journal of Radiology, 85(1011), 197-207. doi:10.1259/bjr/47213729.

[5] Phi, X.-A., Houssami, N., Hooning, M. J., Riedl, C. C., Leach, M. O., Sardanelli, F., ... de Bock, G. H. (2017). Accuracy of screening women at familial risk of breast cancer without a known gene mutation: Individual patient data meta-analysis. European Journal of Cancer, 85, 31-38. doi:10.1016/j.ejca.2017.07.055.

[6] Morris, E.A., Comstock, C.E., Lee, C.H. (2013). ACR BI-RADS Magnetic Resonance Imaging, in: D’Orsi, C.J., Sickles, E.A., Mendelson, E.B., Morris, E.A. (eds.). ACR BI-RADS Atlas, Breast Imaging Reporting and Data System. Reston, VA, American College of Radiology. 
[7] Mahoney, M. C., Gatsonis, C., Hanna, L., DeMartini, W. B., \& Lehman, C. (2012). Positive Predictive Value of BI-RADS MR Imaging. Radiology, 264(1), 51-58. doi:10.1148/radiol.12110619.

[8] Ballesio, L., Di Pastena, F., Gigli, S., D’Ambrosio, I., Aceti, A., Pontico, M., Manganaro, L., Porfiri, L.M., Tardioli, S. (2014). Non mass-like enhancement categories detected by breast MRI and histological findings. Eur Rev Med Pharmalcol Sci 18, 910917. PMID: 24706319.

[9] Shao, Z., Wang, H., Li, X., Liu, P., Zhang, S., \& Cao, S. (2013). Morphological Distribution and Internal Enhancement Architecture of Contrast-Enhanced Magnetic Resonance Imaging in the Diagnosis of Non-Mass-Like Breast Lesions: A MetaAnalysis. The Breast Journal, 19(3), 259-268. doi:10.1111/tbj.12101.

[10] Wilhelm, A., McDonough, M. D., \& DePeri, E. R. (2012). Malignancy Rates of Non-masslike Enhancement on Breast Magnetic Resonance Imaging Using American College of Radiology Breast Imaging Reporting and Data System Descriptors. The Breast Journal, 18(6), 523-526. doi:10.1111/tbj.12008.

[11] Bartella, L., Liberman, L., Morris, E. A., \& Dershaw, D. D. (2006). Nonpalpable Mammographically Occult Invasive Breast Cancers Detected by MRI. American Journal of Roentgenology, 186(3), 865-870. doi:10.2214/ajr.04.1777.

[12] Baltzer, P. A. T., Benndorf, M., Dietzel, M., Gajda, M., Runnebaum, I. B., \& Kaiser, W. A. (2010). False-Positive Findings at Contrast-Enhanced Breast MRI: A BI-RADS Descriptor Study. American Journal of Roentgenology, 194(6), $1658-1663$. doi:10.2214/ajr.09.3486.

[13] El Khoury, M., Lalonde, L., David, J., Labelle, M., Mesurolle, B., \& Trop, I. (2015). Breast imaging reporting and data system (BI-RADS) lexicon for breast MRI: Interobserver variability in the description and assignment of BI-RADS category. European Journal of Radiology, 84(1), 71-76. doi:10.1016/j.ejrad.2014.10.003.

[14] Gutierrez, R. L., DeMartini, W. B., Eby, P. R., Kurland, B. F., Peacock, S., \& Lehman, C. D. (2009). BI-RADS Lesion Characteristics Predict Likelihood of Malignancy in Breast MRI for Masses But Not for Nonmasslike Enhancement. American Journal of Roentgenology, 193(4), 994-1000. doi:10.2214/ajr.08.1983.

[15] Kul, S., Eyuboglu, I., Cansu, A., \& Alhan, E. (2013). Diagnostic efficacy of the diffusion weighted imaging in the characterization of different types of breast lesions. Journal of Magnetic Resonance Imaging, 40(5), 1158-1164. doi:10.1002/jmri.24491

[16] Imamura, T., Isomoto, I., Sueyoshi, E., Yano, H., Uga, T., Abe, K., ... Uetani, M. (2010). Diagnostic Performance of ADC for Non-mass-like Breast Lesions on MR Imaging. Magnetic Resonance in Medical Sciences, 9(4), 217-225. doi:10.2463/mrms.9.217.

[17] Partridge, S. C., Mullins, C. D., Kurland, B. F., Allain, M. D., DeMartini, W. B., Eby, P. R., \& Lehman, C. D. (2010). Apparent Diffusion Coefficient Values for Discriminating Benign and Malignant Breast MRI Lesions: Effects of Lesion Type and Size. American Journal of Roentgenology, 194(6), 1664-1673. doi:10.2214/ajr.09.3534.

[18] Yabuuchi, H., Matsuo, Y., Kamitani, T., Setoguchi, T., Okafuji, T., Soeda, H., ... Honda, H. (2010). Non-mass-like enhancement on contrast-enhanced breast MR imaging: Lesion characterization using combination of dynamic contrastenhanced and diffusion-weighted MR images. European Journal of Radiology, 75(1), e126-e132. doi:10.1016/j.ejrad.2009.09.013.

[19] Liberman, L., Morris, E. A., Lee, M. J.-Y., Kaplan, J. B., LaTrenta, L. R., Menell, J. H., ... Dershaw, D. D. (2002). Breast Lesions Detected on MR Imaging: Features and Positive Predictive Value. American Journal of Roentgenology, 179(1), 171178. doi:10.2214/ajr.179.1.1790171.

[20] Tozaki, M., \& Fukuda, K. (2006). High-Spatial-Resolution MRI of Non-Masslike Breast Lesions: Interpretation Model Based on BI-RADS MRI Descriptors. American Journal of Roentgenology, 187(2), 330-337. doi:10.2214/ajr.05.0998.

[21] Chikarmane, S. A., Michaels, A. Y., \& Giess, C. S. (2017). Revisiting Nonmass Enhancement in Breast MRI: Analysis of Outcomes and Follow-Up Using the Updated BI-RADS Atlas. American Journal of Roentgenology, 209(5), 1178-1184. doi:10.2214/ajr.17.18086.

[22] Uematsu, T., \& Kasami, M. (2012). High-Spatial-Resolution 3-T Breast MRI of Nonmasslike Enhancement Lesions: An Analysis of Their Features as Significant Predictors of Malignancy. American Journal of Roentgenology, 198(5), $1223-1230$. doi:10.2214/ajr.11.7350.

[23] Sakamoto, N., Tozaki, M., Higa, K., Tsunoda, Y., Ogawa, T., Abe, S., ... Fukuma, E. (2008). Categorization of non-mass-like breast lesions detected by MRI. Breast Cancer, 15(3), 241-246. doi:10.1007/s12282-007-0028-6.

[24] Tozaki, M., Igarashi, T., \& Fukuda, K. (2006). Breast MRI Using the VIBE Sequence: Clustered Ring Enhancement in the Differential Diagnosis of Lesions Showing Non-Masslike Enhancement. American Journal of Roentgenology, 187(2), 313321. doi:10.2214/ajr.05.0881. 
[25] Grimm, L. J., Anderson, A. L., Baker, J. A., Johnson, K. S., Walsh, R., Yoon, S. C., \& Ghate, S. V. (2015). Interobserver Variability Between Breast Imagers Using the Fifth Edition of the BI-RADS MRI Lexicon. American Journal of Roentgenology, 204(5), 1120-1124. doi:10.2214/ajr.14.13047.

[26] Chen, X., Li, W., Zhang, Y., Wu, Q., Guo, Y., \& Bai, Z. (2010). Meta-analysis of quantitative diffusion-weighted MR imaging in the differential diagnosis of breast lesions. BMC Cancer, 10(1). doi:10.1186/1471-2407-10-693.

[27] Bickel, H., Pinker, K., Polanec, S., Magometschnigg, H., Wengert, G., Spick, C., ... Baltzer, P. (2016). Diffusion-weighted imaging of breast lesions: Region-of-interest placement and different ADC parameters influence apparent diffusion coefficient values. European Radiology, 27(5), 1883-1892. doi:10.1007/s00330-016-4564-3.

[28] Arponent, O., Sudah, M., Masarwah, A., Taina, M., Rautiainen, S., Könönen, M., Sironen, R., Kosma, V.M., Sutela, A., Hakumäki, J., Vanninen, R. (2015). Correction: Diffusion-Weighted Imaging in 3.0 Tesla Breast MRI: Diagnostic Performance and Tumor Characterization Using Small Subregions vs. Whole Tumor Regions of Interest. PLOS ONE, 10(10), e0141833. doi:10.1371/journal.pone.0141833.

[29] Min, Q., Shao, K., Zhai, L., Liu, W., Zhu, C., Yuan, L., \& Yang, J. (2015). Differential diagnosis of benign and malignant breast masses using diffusion-weighted magnetic resonance imaging. World Journal of Surgical Oncology, $13(1), 32$. doi:10.1186/s12957-014-0431-3.

[30] Bogner, W., Pinker-Domenig, K., Bickel, H., Chmelik, M., Weber, M., Helbich, T. H., ... Gruber, S. (2012). Readoutsegmented Echo-planar Imaging Improves the Diagnostic Performance of Diffusion-weighted MR Breast Examinations at 3.0 T. Radiology, 263(1), 64-76. doi:10.1148/radiol.12111494.

[31] Nogueira, L., Brandão, S., Matos, E., Nunes, R. G., Ferreira, H. A., Loureiro, J., \& Ramos, I. (2015). Region of interest demarcation for quantification of the apparent diffusion coefficient in breast lesions and its interobserver variability. Diagnostic and Interventional Radiology, 21(2), 123-127. doi:10.5152/dir.2014.14217. 